\title{
Implementation of a New Hybrid \\ Methodology for Fault Signal Classification Using Short -Time Fourier Transform and Support Vector Machines
}

Tribeni Prasad Banerjee, Swagatam Das, Joydeb Roychoudhury, and Ajith Abraham

\begin{abstract}
Increasing the safety of a high-speed motor used in aerospace application is a critical issue. So an intelligent fault aware control methodology is highly research motivated area, which can effectively identify the early fault of a motor from its signal characteristics. The signal classification and the control strategy with a hybrid technique are proposed in this paper. This classifier can classify the original signal and the fault signal. The performance of the system is validated by applying the system to induction motor faults diagnosis. According to our experiments in BLDC motor controller results, the system has potential to serve as an intelligent fault diagnosis system in other hard real time system application. To make the system more robust we make the controller more adaptive that give the system response more reliable.
\end{abstract}

Keywords: Real time system, Support Vector Machine, Sort Time Fourier Transform, embedded system, Brash less Direct Current Motor, Intelligent interactive control, supervisory control, signal classification, Fault Classifier, Mechatronics, fault diagnosis.

Tribeni Prasad Banerjee · Joydeb Roychoudhury

Embedded System Laboratory, Central Mechanical Engineering Research Institute,

Durgapur-713209, India

e-mail: t_p_banerjee@yahoo.com, jrc@cmeri.res.in

Swagatam Das

Electronics and Telecommunication Engineering Department Jadavpur University,

Jadavpur-700035, India

e-mail: swagatamdas19@yahoo.co.in

Ajith Abraham

Machine Intelligence Research Labs (MIR Labs), Scientific Network for

Innovation and Research Excellence, USA

e-mail: ajith.abrahamaieee.org

E. Corchado et al. (Eds.): SOCO 2010, AISC 73, pp. $219-225$.

springerlink.com (C) Springer-Verlag Berlin Heidelberg 2010 


\section{Introduction}

Fault diagnosis is very important for safe operation and preventing rescue [1]. It is preferable to find fault before complete system failure. A system with incipient fault but it will lead to a system to a catastrophic failure causing downtime and large losses. Fault diagnostics is a kind of signal classification as far as its essence is concerned. Support vector machines (SVM) have a good generalization capability even in the small-sample cases of classification and have been successfully applied in fault detection and diagnosis [2][3]. SVMs [4-7] provide efficient and powerful classification algorithms that are capable of dealing with high-dimensional input features and with theoretical bounds on the generalization error and sparseness of the solution provided by statistical learning theory [4]. Classifiers based on SVMs have few free parameters requiring tuning, are simple to implement, and are trained through optimization of a convex quadratic cost function, which ensures the uniqueness of the SVM solution. Furthermore, SVM-based solutions are sparse in the training data and are defined only by the most "informative" training points.

In control and automata theory, a state is defined as a condition. That characterizes a prescribed relationship of input, output and input to the next state. Thus knowledge of the state of a system gives better understanding through observation and we can subsequently take necessary steps for controlling the system, for example, stabilizing a system using state feedback. This implies that the current state of a system determines the future plan of action. So it becomes extremely important to devise some methodology by which the state of a system can be observed correctly within a stipulated time frame. For example a particular state of a feedback control system cannot be observed directly due to its own compensator which is one of the limitations of the feedback controller. A separate hardware with very low observation latency period [6] is necessary to observe this unobserved state which is called a sub state or internal state directly related to the health of the system. In order to make system fault aware a sub state observer is conceived whose execution time of the event sequences (necessary for sub state observation) is much faster and due to this high speed execution and subsequent scheduling technique, it can observe the sub state behavior of a system and thus differentiate between two almost similar states with different end effects.

In this paper, we proposed an incipient signal classification which is optimally separated by SVM and after classification of the signal or a optimal threshold value a controller strategy is implanted into a evolvable hardware which gives a hard and real time responsive or responsive system. 


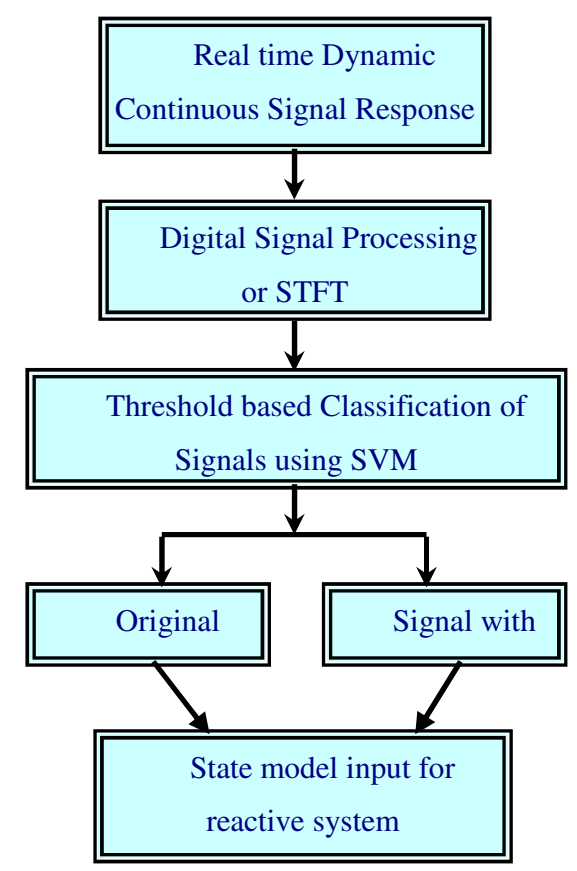

Fig. 1 A generic Scheme for signal classification and reactive system and their consequences

The basic scheme of Signal classification is illustrated in figure 1.

\section{Principal of Operation Signal Classification}

\subsection{Real Time Dynamic Continuous Signal Response}

Hard real time system is that system which has a critical time limit [10] or a reactive system where the system inputs are dynamically changes with time. Within that time duration the system has to response otherwise the system is going to a catastrophic loss or high probability for human death. The real time systems are basically two types one is reactive system and another is embedded system. A reactive system is always react with the environment (online aircraft valve signal monitoring from a actuator signal) and another is embedded system which is used to control specialized hardware that is installed within a larger system (such as a microprocessor that controls anti-lock brakes in an automobile) In our system is more reactive with the environments. Here in our proposed system the online dynamic continuous signals is comes and we capture the signal and transform it and pass through into our classifier and take the decision. The EFSM model [6] does the real time operation within a few micro seconds. This makes the system first 
responsive according to the decision of the output and the reactive system makes a more intelligent neuro adaptive system.

\subsection{Signal Preprocessing for STFT}

Signal classification is a major research area in the real time DSP, because of the real time operation the classification of signal is very crucial issue. Even the signal classification has lots of problem, so we prefer the STFT. Here we briefly review the chaos detector based on the STFT proposed in [9-10]. The short-time Fourier transform (STFT), or alternatively short-term Fourier transform, is a Fourier-related transform used to determine the sinusoidal frequency and phase content of local sections of a signal as it changes over time.

$$
\operatorname{STFT}(t, f)=\int_{-\alpha}^{\alpha} x(t+\tau) w(\tau) e^{-j 2 \Pi f \tau} d \tau
$$

Where $\mathrm{x}(\mathrm{t})$ is signal of interest (in this paper, it is a voltage or a current from the BLDC motor), and $\mathrm{w}(\tau)$ is the window function, where $\mathrm{w}(\tau)=0$ for $|\tau|>\mathrm{T} / 2$ and $\mathrm{T}$ is window width. In order to avoid complex-valued STFT, we use its squared magnitude, i.e., the spectrogram $S P E C(t, f)=|5 T E T(t \times f)|^{2}$. As shown in figure 2

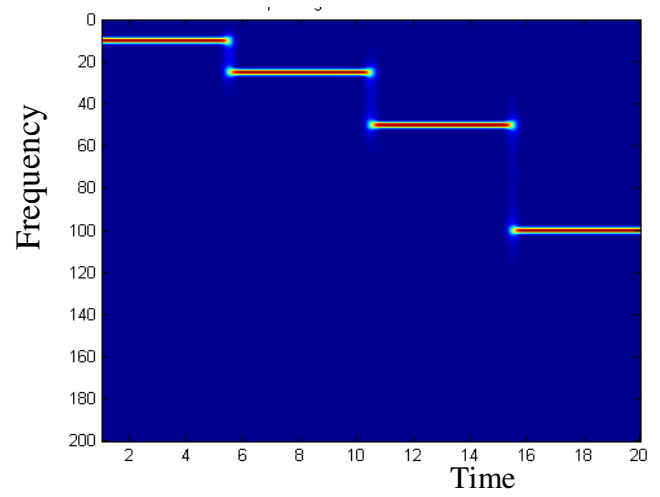

Fig. 2 Original Signal after the STFT transformed

\subsection{Signal Classification of Using SVM}

Support vector machines (SVM) [10] have been widely used in the fault classification and diagnosis of machines in the past few years, especially in the fault diagnosis [11], for dynamic procedures such as the starting, the stopping, and the changing of working mode. SVM certainly outperform some other artificial intelligence methods because it is able to maximize the generalization performance 
of a trained classifier. This may not be easily achieved by using HMM or ANN, because ANN are prone to having a higher specificity and a lower sensitivity and HMM are prone to having a higher sensitivity and a lower specificity. SVM aims at the optimal solution in the available information rather than just the optimal solution when the sample number tends towards infinitely large. It has a good generalization when the samples are few, so it is especially fit for classification, forecasting and estimation in small-sample cases such as fault diagnosis, in most cases whose bottleneck problem is the lack of fault samples.

Once the frequency is separated by STFT technique, then the signals are taken into the classification stage, here according to the maximized threshold value are calculated by SVM. Because that optimum threshold value is given to the state machine for taken into the real time decision processes. Depending upon the magnitude value the threshold values are set to a specific signal and the different signal magnitude are set to a different class. A signal which is exceed a specific threshold value it is classified as high risk and other which are not exceed is fallen into other class. This threshold value is set dynamically and depending upon the timed model components is already classified form the inputs.

SVM [10], as well as neural networks, have been extensively employed to solve classification problems due to the excellent generalization performance on a wide range problem. In machine fault diagnosis, some researchers have employed SVM as a tool for classification of faults. For example, ball bearing faults [9], gear faults [10], condition classification of small reciprocating compressor, and induction motor [11]. In this paper, we also proposed a incipient faults identification in dynamic signal diagnosis system of induction motor based on start-up transient current signal using component analysis and SVM.

In SVM, the original input space is mapped into a high-dimensional dot product space called a feature space, and in the feature space. SVM' s have the potential to handle very large feature spaces, because the training of SVM is carried out so that the dimension of classified vectors does not have as a distinct influence on the performance of SVM as it has on the performance of conventional classifiers. That is why it is noticed to be especially efficient in large classification problems [12].This will also benefit in fault classification, because the number of features to be the basis of fault diagnosis may not have to be limited. Also, SVM-based classifiers are claimed to have good generalization properties compared to conventional classifiers, because in training the SVM classifier, the so-called structural misclassification risk is to be minimized, whereas traditional classifiers are usually trained so that the empirical risk is minimized. When a signal falls outside the clusters, it is tagged as a potential motor failure [13]. Since a fault condition is not a spurious event it can degrade the system, the postprocessor alarms the user only after multiple indications of a potential failure have occurred. In this way, the time duration modeling of the State machine is incorporated into the monitoring system and protects the reactive system by alarming on random signals that have been identified as in our system from the mixed signal the signal of interest has been separated from the injected fault signal, shown in the figure 3 (a) $\&(b)$. 


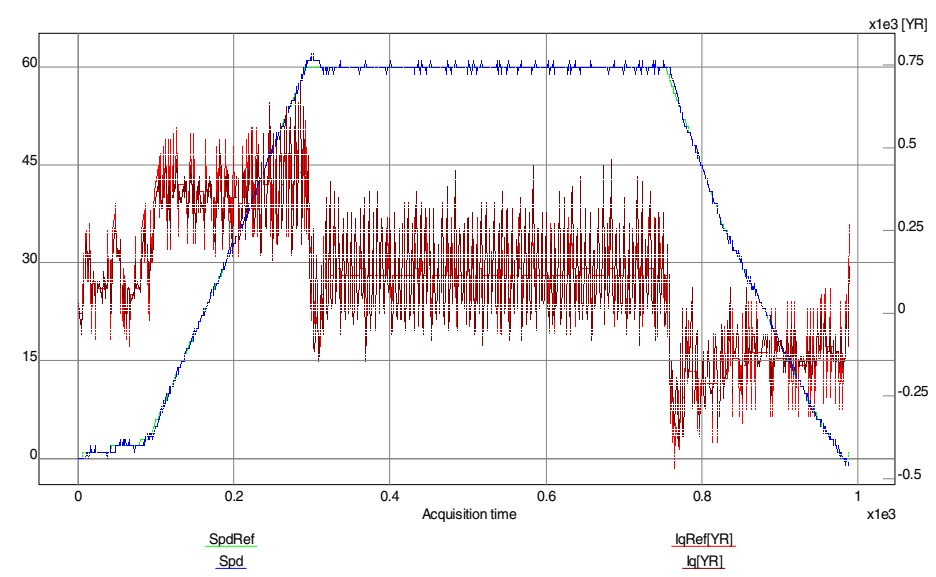

Fig 3. Original Signal and the fault signal are both before the system input

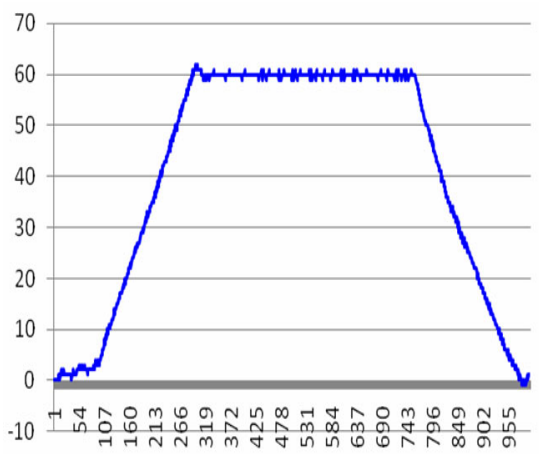

(a)

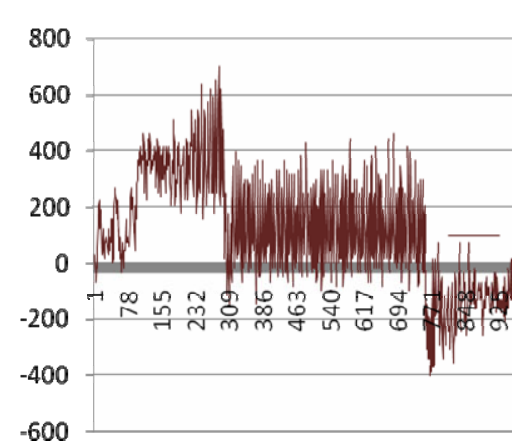

(b

Fig. 3 The actual signal $(a, b)$ and injected fault signal after separation

\section{Experimental Set Up and System Description}

We used the TMS120F2812, which is a 12-bit Fixed-point DSP of Harvard Bus Architecture. It has separated Data Bus and Program Bus. It also supports atomic operations, $12 \times 12$ bit MAC operations and fast interrupts response. It is able to control the motor easily with TMS120F2812 because it has two event managers, which include capture function, PWM function, and QEP (Quadrature -Encoder Pulse) function. Since it also supports variable serial port peripherals such as CAN, SCI, and SPI[16], it is possible to communicate with external device and exchange control signal and data. The prototype is designed with a control circuit based on the "MSK2812 Kit C Pro VS" (from Technosoft S.A.). Operating at a 190-MHz frequency, with double event-manager signals, increased internal memory, etc., this new kit offers all features needed for an advanced digital control implementation. All communication between the PC and the DSP board is done through the RS-192 interface using a real-time serial communication monitor with download/upload 
functions, debug and inspect facilities [14, 15]. PROCEV28x, graphical evaluation/analysis software for the specific peripherals is embedded in the TMS120F2812 DSP.

\section{Conclusions}

The experiments are done in the laboratory. In our proposed system the intelligent signal classifier classifies the original signal from the fault signal. At the first level, the classifier separates the signal by STFT methods, and the time duration based model classified the signal very smoothly as depicted in Figure 3. We believe that the system has potential to serve an intelligent fault diagnosis system in other hard real time system applications also.

\section{References}

[1] Yongming, Y., Bin, W.: A review on induction motor online fault diagnosis. In: The 3rd International Power Electronic And Motion Control Conference 2000 (IPEMC), pp. 1353-1358. IEEE, Los Alamitos (2000)

[2] Matthias, P., Stefan, O., Manfred, G.: Support Vector Approaches for Engine Knock Detection. In: International Joint Conference on Neural Networks, pp. 969-974. IEEE Press, Washington (1999)

[3] Chiang, L.H., Kotanchek, M.E., Kordon, A.K.: Fault diagnosis based on fisher discriminate analysis and support vector machines. Computers and Chemical Engineering 28, 1389-1401 (2004)

[4] Scholkopf, B., Smola, A.: Learning With Kernels. MIT Press, Cambridge (2002)

[5] Herbrich, R.: Learning Kernel Classifiers: Theory and Algorithms. MIT Press, Cambridge (2002)

[6] Mall, R.: Real time systems. In: Theory and practice, Pearson Publication, London (2007)

[7] Alur, R., Dill, D.: The Theory of Timed Automata. Theoretical Computer Science 120, 143-235 (1994)

[8] Jack, L.B., Nandi, A.K.: Fault detection using support vector machines and artificial neural network, augmented by genetic algorithms. Mechanical System Signal Process 14, 373-390 (2002)

[9] Boashash, B. (ed.): Time frequency Signal Analysis and Applications. Elsevier, Amsterdam (2003)

[10] Vapnik, V.N.: The nature of statistical learning theory. Springer, New York (1999)

[11] Yang, B.S., et al.: Fault diagnosis of rotating machinery based on multi-class support vector machines. Journal Mechanical Science Technology 19, 845-858 (2005)

[12] Yang, B.S., Hwang, W.W., Kim, D.J., Tan, A.C.: Condition classification of small reciprocating compressor for refrigerators using artificial neural networks and support vector machines. Mechanical System Signal Process 19, 371-390 (2005)

[13] Ma, X.X., Huang, X.Y., Chai, Y.: PTMC classification algorithm based on support vector machines and its application to fault diagnosis. Control and Decision 14, 212-284 (2003) (in Chinese)

[14] Texas Instruments, TMS120F2812 Digital Signal Processors Data Manual (2005)

[15] Texas Instruments, TMS120F28x DSP Enhanced Controller Area Network (e CAN) Reference Guide (2005) 